\title{
Orbits of Free Cyclic Submodules Over Rings of Lower Triangular Matrices
}

\author{
Edyta Bartnicka@
}

\begin{abstract}
Given a ring $T_{n}(n \geqslant 2)$ of lower triangular $n \times n$ matrices with entries from an arbitrary field $F$, we completely classify the orbits of free cyclic submodules of ${ }^{2} T_{n}$ under the action of the general linear group $G L_{2}\left(T_{n}\right)$. Interestingly, the total number of such orbits is found to be equal to the Bell number $B_{n}$. A representative of each orbit is also given.
\end{abstract}

Mathematics Subject Classification. 15B33, 16D40.

Keywords. Orbits of submodules, free cyclic submodules, associative rings with unity, lower triangular matrices.

\section{Introduction}

The general linear group $G L_{2}(R)$ acts in natural way on the set of all free cyclic submodules of the two-dimensional left free module ${ }^{2} R$. In the case of an arbitrary associative ring $R$ with unity, $G L_{2}(R)$-orbit of free cyclic submodules generated by unimodular pairs is the projective line $\mathbb{P}(R)$, which has already been discussed in a number of papers (see, e.g., [2-4]). We also refer to $[5,7]$ for the definition of the unimodularity. There also exist rings featuring nonunimodular free cyclic submodules. In this note we investigate the case of a ring $T_{n}$ of lower triangular $n \times n$ matrices $A=\left[\begin{array}{cccc}a_{11} & 0 & \ldots & 0 \\ a_{21} & a_{22} & \ldots & 0 \\ \vdots & \vdots & \ddots & \vdots \\ a_{n 1} & a_{n 2} & \ldots & a_{n n}\end{array}\right]$ over a fixed field $F$, where $n \geqslant 2$. 
The main motivation for this paper is the work of Havlicek and Saniga [6], where a thorough classification of the vectors of the free left module ${ }^{2} T_{2}$ over a ring of ternions $T_{2}$ up to the natural action of $G L_{2}\left(T_{2}\right)$ was given; it was found that there exactly two (one unimodular and one non-unimodular) orbits of free cyclic submodules of ${ }^{2} T_{2}$ under the action of $G L_{2}\left(T_{2}\right)$.

In [1] there are described all orbits of free cyclic submodules in the $\operatorname{ring} T_{3}$ under the action of $G L_{2}\left(T_{3}\right)$. Here we extend and generalize the findings of [1] to an arbitrary ring $T_{n}$ and provide the full classification of orbits of free cyclic submodules of ${ }^{2} T_{n}$. The starting point of our analysis is a complete characterization of free cyclic submodules, unimodular pairs and outliers generating free cyclic submodules in the case of $T_{n}$. Next, we will introduce representatives of all $G L_{2}\left(T_{n}\right)$-orbits (Theorems 1 and 2 ). Finally, we will prove that the total number of $G L_{2}\left(T_{n}\right)$-orbits is equal to the Bell number $B_{n}$ (Theorem 3).

\section{Free Cyclic Submodules $T_{n}(A, B)$}

Consider the free left module ${ }^{2} R$ over a ring $R$ with unity. Let $(a, b) \subset{ }^{2} R$, then the set $R(a, b)=\{r(a, b) ; r \in R\}$ is a left cyclic submodule of ${ }^{2} R$. If the equation $(r a, r b)=(0,0)$ implies that $r=0$, then $R(a, b)$ is called free. We give now the condition for a cyclic submodule $M_{n}(A, B) \subset{ }^{2} M_{n}$, where $M_{n}$ is the ring of $n \times n$ matrices over a fixed field $F$, to be free.

Lemma 1. A cyclic submodule $M_{n}(A, B)$ is free if, and only if, the rank of $[A \mid B]$ is $n$.

Proof. Let $k_{j}$ be the $j$-th column of $[A \mid B]$ and let $w_{i}=\left[x_{i 1} x_{i 2} \ldots x_{i n}\right], i=$ $1,2, \ldots, n$.

Then $[A \mid B]=\left[k_{1} k_{2} \ldots k_{2 n}\right]$ and $X=\left[\begin{array}{cccc}x_{11} & x_{12} & \ldots & x_{1 n} \\ x_{21} & x_{22} & \ldots & x_{2 n} \\ \vdots & \vdots & \vdots & \\ x_{n 1} & x_{n 2} & \ldots & x_{n n}\end{array}\right]=\left[\begin{array}{c}w_{1} \\ w_{2} \\ \vdots \\ w_{n}\end{array}\right]$.

" $\Leftarrow$ " Suppose that the rank of $[A \mid B]$ is $n$. Equivalently, exactly $n$ columns $k_{j}$ of $[A \mid B]$ represent a set of linearly independent vectors. Then the equation

$$
\left[\begin{array}{c}
w_{1} \\
w_{2} \\
\vdots \\
w_{n}
\end{array}\right]\left[k_{1} k_{2} \ldots k_{2 n}\right]=0
$$

(where 0 denotes the zero matrix of size $n \times 2 n$ ) implies that linear functionals $w_{1}, w_{2}, \ldots, w_{n}: F^{n} \rightarrow F$ are zeros on $n$ linearly independent vectors of the space $F^{n}$. So they are zero and hence $M_{n}(A, B)$ is free.

" $\Rightarrow$ " Assume that a submodule $M_{n}(A, B)$ is free. Then the fact that any set of linear functionals $w_{1}, w_{2}, \ldots, w_{n}: F^{n} \rightarrow F$ are zeros on vectors $k_{1}, k_{2}, \ldots, k_{2 n}$ 
implies $w_{1}=w_{2}=\ldots=w_{n}=0$. Consequently, there exists a set of $n$ linearly independent vectors $k_{j}$ and so the rank of $[A \mid B]$ is $n$.

Remark 1. The statement of Lemma 1 remains true if one replaces the ring $M_{n}$ by any of its subrings, in particular by the ring $T_{n}$.

It is well known that any unimodular pair generates a free cyclic submodule; we shall call such free cyclic submodules unimodular. Now, we will introduce the condition of unimodularity in the case of $T_{n}$.

Remark 2. A pair $(A, B) \subset{ }^{2} T_{n}$ is unimodular if, and only if, $a_{i i} \neq 0 \vee b_{i i} \neq 0$ for any $i=1,2, \ldots, n$.

Proof. According to the general definition of unimodularity, a pair $(A, B) \subset$ ${ }^{2} T_{n}$ is unimodular if there exist matrices $X, Y \in T_{n}$ such that $A X+B Y=$ $U \in T_{n}^{*}$, where $T_{n}^{*}$ denotes the group of invertible elements of the ring $T_{n}$. As

$$
A X+B Y=\left[\begin{array}{ccc}
a_{11} x_{11}+b_{11} y_{11} & & 0 \\
& \ddots & \\
* & & a_{n n} x_{22}+b_{n n} y_{n n}
\end{array}\right]
$$

for any $X, Y \in T_{n}(q)$, then $A X+B Y=U \Leftrightarrow a_{i i} x_{i i}+b_{i i} y_{i i} \neq 0$ for any $i=1,2, \ldots, n$. This is equivalent to $a_{i i} x_{i i} \neq-b_{i i} y_{i i}$ for any $i=1,2, \ldots, n$. Hence $(A, B) \subset{ }^{2} T_{n}$ is unimodular if, and only if, $a_{i i} \neq 0 \vee b_{i i} \neq 0$ for any $i=1,2, \ldots, n$.

Another type of free cyclic submodules is the one represented by pairs that are not contained in any cyclic submodule generated by a unimodular pair, so-called outliers (first introduced in [8]). As it was pointed out in $[1,6]$, the number of such non-unimodular free cyclic submodules can be quite large although, of course, not all outliers generate free cyclic submodules.

Lemma 2. A pair $(A, B) \subset{ }^{2} T_{n}$ is an outlier generating a free cyclic submodule if, and only if, $(A, B)$ is non-unimodular and $\operatorname{rank}[A \mid B]=n$.

Proof. According to $\left[1\right.$, Theorem 1(2)] if a non-unimodular pair $(A, B) \subset{ }^{2} T_{n}$ generates a free cyclic submodule, then $(A, B)$ is an outlier. Lemma 1 and Remark 1 then yield the desired claim.

It also follows that there are no other free cyclic submodules $T_{n}(A, B)$ apart from those generated by unimodular pairs or outliers. Moreover, a cyclic submodule $T_{n}(A, B)$ generated by a unimodular pair cannot have non-unimodular generators (see [1, Corollary 1]).

\section{Representatives of $G L_{2}\left(T_{n}\right)$-Orbits}

The general linear group $G L_{2}\left(T_{n}\right)$ acts in a natural way (from the right) on the free left $T_{n}$-module. Orbits of the set of all free cyclic submodules 
$T_{n}(q)(A, B) \subset{ }^{2} T_{n}(q)$ under the action of $G L_{2}\left(T_{n}\right)$ are called $G L_{2}\left(T_{n}\right)$-orbits. To show the explicit form of representatives of such orbits, we will use the following remark:

Remark 3. Let $X, Y, W, Z \in T_{n}$. The matrix $\left[\begin{array}{cc}X & Y \\ W & Z\end{array}\right]$ is invertible, i.e. it is an element of the group $G L_{2}\left(T_{n}\right)$, if, and only if, $x_{i i} z_{i i}-y_{i i} w_{i i} \neq 0$ for all $i=1,2, \ldots, n$.

Let (A, B) be a pair of ${ }^{2} T_{n}$ such that for all $i=1,2, \ldots, n, j=1,2, \ldots, i-1$ :

- $a_{i i}, b_{i j} \in\{0,1\}$,

- $a_{i j}=b_{i i}=0$,

- the number of non-zero entries of $[\mathrm{A} \mid \mathrm{B}]$ is equal to its rank and it is $n$.

We will use the grotesk typeface for such pairs. According to Lemma 1 and Remark 1 any pair $(\mathrm{A}, \mathrm{B}) \in{ }^{2} T_{n}$ generates a free cyclic submodule.

Theorem 1. Let $(\mathrm{A}, \mathrm{B}),(\mathrm{C}, \mathrm{D}) \in{ }^{2} T_{n}$. Submodules $T_{n}(\mathrm{~A}, \mathrm{~B}), T_{n}(\mathrm{C}, \mathrm{D})$ are representatives of distinct $G L_{2}\left(T_{n}\right)$-orbits if, and only if, $(\mathrm{A}, \mathrm{B}) \neq(\mathrm{C}, \mathrm{D})$.

Proof. " $\Rightarrow$ " This is obvious.

"६" We give the proof by contradiction. Assume that $T_{n}(\mathrm{~A}, \mathrm{~B}), T_{n}(\mathrm{C}, \mathrm{D})$ are in the same $G L_{2}\left(T_{n}\right)$-orbit, i.e. there exists $\left[\begin{array}{ll}X & Y \\ W & Z\end{array}\right] \in G L_{2}\left(T_{n}\right)$ such that $(\mathrm{A}, \mathrm{B})\left[\begin{array}{cc}X & Y \\ W & Z\end{array}\right]=(\mathrm{C}, \mathrm{D})$. Hence,

$$
\left\{\begin{array}{l}
a_{i i} x_{i i}=c_{i i} \\
a_{i i} y_{i i}=0
\end{array}\right.
$$

for any $i \in\{1,2, \ldots, n\}$.

Submodules $T_{n}(\mathrm{~A}, \mathrm{~B}), T_{n}(\mathrm{C}, \mathrm{D})$ are free, thus $a_{11}, c_{11} \in F^{*}=F \backslash\{0\}$, and so $a_{11}=c_{11}=1$.

Let $i \in\{2,3, \ldots, n\}$. Consider two cases:

1. $a_{i i}=1$; Then by the above system of equations and according to Remark 3 we get $y_{i i}=0, x_{i i} \neq 0$ and $c_{i i} \neq 0$. Consequently, $c_{i i}=a_{i i}=1$ and so all the remaining entries in the $i$-th row of matrices A, B, C, D are equal to zero.

2. $a_{i i}=0$; Then we get immediately $c_{i i}=a_{i i}=0$. The form of $(\mathrm{A}, \mathrm{B}) \in{ }^{2} T_{n}$ implies that there exists exactly one non-zero entry $b_{i j}$ in the $i$-th row of the matrix B. Suppose then that $b_{i j}=1$ for some $j \in\{1,2, \ldots, i-1\}$. We obtain the following system of equations:

$$
\left\{\begin{array}{l}
c_{i j}=0=a_{i i} x_{i j}+b_{i j} w_{j j}+b_{i(j+1)} w_{(j+1) j}+\ldots+b_{i(i-1)} w_{(i-1) j}=b_{i j} w_{j j}, \\
d_{i j}=a_{i i} y_{i j}+b_{i j} z_{j j}+b_{i(j+1)} z_{(j+1) j}+\ldots+b_{i(i-1)} z_{(i-1) j}=b_{i j} z_{j j} .
\end{array}\right.
$$


Therefore $w_{j j}=0$. Remark 3 leads to $z_{j j} \neq 0$, and so $d_{i j} \neq 0$. Thus $d_{i j}=b_{i j}=1$ and all the remaining entries in the $i$-th row of matrices A, B, C, D are equal to zero.

This means that $(\mathrm{A}, \mathrm{B})=(\mathrm{C}, \mathrm{D})$, which completes the proof.

Theorem 2. Any $G L_{2}\left(T_{n}\right)$-orbit has a unique representative $T_{n}(\mathrm{~A}, \mathrm{~B}) \subset{ }^{2} T_{n}$.

Proof. Taking into account Theorem 1 it suffices to show that any $G L_{2}\left(T_{n}\right)$ orbit has a representative of the form $T_{n}(\mathrm{~A}, \mathrm{~B}) \subset{ }^{2} T_{n}$. This is equivalent to saying that for any free cyclic submodule $T_{n}(A, B) \subset{ }^{2} T_{n}$ there exist matrices $Q \in G L_{2}\left(T_{n}\right)$ and $U \in T_{n}^{*}$ such that $U(A, B) Q=(\mathrm{A}, \mathrm{B})$. We will now show that this indeed holds.

Let $T_{n}(A, B)$ be any free cyclic submodule of ${ }^{2} T_{n}$. We multiply $(A, B) \in{ }^{2} T_{n}$ by $\left[\begin{array}{ll}X & Y \\ W & Z\end{array}\right] \in G L_{2}\left(T_{n}\right)$, with entries $y_{i i}=-a_{i i}^{-1} b_{i i} z_{i i}$ if $a_{i i} \neq 0$, and $z_{i i}=0$ if $a_{i i}=0, i=1,2, \ldots, n$. This yields

$$
(A, B)\left[\begin{array}{ll}
X & Y \\
W & Z
\end{array}\right]=(C, D),
$$

where $d_{i i}=0$ for any $i=1,2, \ldots, n$.

The next step is multiplying

$$
P^{-1}(C, D)\left[\begin{array}{cc}
P & 0 \\
0 & I
\end{array}\right]=\left(P^{-1} C P, P^{-1} D\right)=(J, E),
$$

where $P \in T_{n}$ and $J$ is a Jordan normal form of $C$ with block matrices $\lambda_{i}$, which are just elements of $F$. Of course, $e_{i i}=0$ for any $i=1,2, \ldots, n$.

Let $J^{\prime}$ be the matrix obtained from $J$ by replacing any $\lambda_{i} \neq 0$ by $\lambda_{i}^{-1}$, and $\lambda_{i}=0$ by 1 . The result of multiplication $J^{\prime}(J, E)=\left(J^{\prime} J, J^{\prime} E\right)$ is a pair $(\mathrm{A}, F)$, where, for any $i=1,2, \ldots, n, a_{i i}=\left\{\begin{array}{l}1, \lambda_{i} \neq 0 \\ 0, \lambda_{i}=0\end{array} \quad\right.$ and $f_{i i}=0$.

We multiply again the last pair $(\mathrm{A}, F)$ by $\left[\begin{array}{cc}I & -F \\ 0 & I\end{array}\right]$ and obtain a pair $(\mathrm{A},-\mathrm{A} F+$ $F)=(\mathrm{A}, G)$, where $g_{i j}=0$ for any $i, j=1,2, \ldots, n$ such that $a_{i i}=1$.

Suppose that $i_{t}, t \in T$ are the numbers of non-zero rows of matrix $G$, where $i_{t}<i_{s}$ if $t<s$. Let now

1. $j_{t}=j_{1}$ be the number of column of $G$ such that $g_{i_{1} j_{1}} \neq 0$ and $g_{i_{1} k}=0$ for any $k>j_{1}$;

2. $j_{t}, t>1$ be the numbers of columns of $G$ which fulfil all the requirements set:

a. $j_{t} \neq j_{r}$ for any $r=1,2, \ldots, t-1$,

b. $g_{i_{t} j_{t}} \neq 0$,

c. $g_{i_{t} k}=0$ for any $k$ satisfying the two conditions: $k>j_{t}$ and $k \neq j_{r}$ for any $r=1,2, \ldots, t-1$, 
d. a sequence of vectors $\left[\begin{array}{c}g_{i_{1} j_{1}} \\ g_{i_{2} j_{1}} \\ \vdots \\ g_{i_{t} j_{1}}\end{array}\right],\left[\begin{array}{c}g_{i_{1} j_{2}} \\ g_{i_{2} j_{2}} \\ \vdots \\ g_{i_{t} j_{2}}\end{array}\right], \ldots,\left[\begin{array}{c}g_{i_{1} j_{t}} \\ g_{i_{2} j_{t}} \\ \vdots \\ g_{i_{t} j_{t}}\end{array}\right]$ is linearly independent (the possibility of choice of such $j_{t}$ is guaranteed because the rank of $G$ is $|T|$ by Lemma 1 and Remark 1).

Consider the matrix $V$ of $T_{n}$ such that

$$
\sum_{\alpha=j_{t}}^{i_{t}-1} g_{i_{t} \alpha} v_{\alpha j_{t}}=1
$$

and

$$
\sum_{\alpha=l}^{i_{t}-1} g_{i_{t} \alpha} v_{\alpha l}=0
$$

for any $t \in T, l=1,2, \ldots, j_{t}-1$.

We prove now that $V$ is invertible, i.e. we show that $v_{j_{t} j_{t}} \neq 0$ for each $j_{t}$ one by one, from the greatest to the smallest, by using the method described below. Let $j_{t_{m}}=\max \left\{j_{t}, t \in T\right\}$ and let $t$ be a fixed element of $T$. Assume that $j_{t_{1}}<j_{t_{2}}<\cdots<j_{t_{m}}$ be all numbers of columns of $G$ greater than $j_{t}$ and meet the above requirements 3 - 3. From the conditions for $V$ we obtain a system of equations:

$$
\left\{\begin{array}{l}
g_{i_{t} j_{t}} v_{j_{t} j_{t}}+g_{i_{t} j_{t_{1}}} v_{j_{t_{1}} j_{t}}+\cdots+g_{i_{t} j_{t_{m}}} v_{j_{t_{m}} j_{t}}=1 \\
g_{i_{t_{1}} j_{t}} v_{j_{t} j_{t}}+g_{i_{t_{1}} j_{t_{1}}} v_{j_{t_{1}} j_{t}}+\cdots+g_{i_{1} j_{t_{m}}} v_{j_{t_{m}} j_{t}}=0 \\
g_{i_{t_{2}} j_{t}} v_{j_{t} j_{t}}+g_{i_{t_{2}} j_{t_{1}}} v_{j_{t_{1}} j_{t}}+\cdots+g_{i_{t_{2}} j_{t_{m}}} v_{j_{t_{m}} j_{t}}=0 \\
\vdots \\
g_{i_{t_{m}} j_{t}} v_{j_{t} j_{t}}+g_{i_{t_{m}} j_{t_{1}}} v_{j_{t_{1}} j_{t}}+\cdots+g_{i_{t_{m}} j_{t_{m}}} v_{j_{t_{m}} j_{t}}=0
\end{array}\right.
$$

Obviously, $v_{j_{t} j_{t}}=g_{i_{t} j_{t}}^{-1}$ if $j_{t}=j_{t_{m}}$, otherwise we solve these equations from the last to the first one for $v_{j_{t_{m}} j_{t}}, v_{j_{t_{m-1}} j_{t}}, \ldots, v_{j_{t_{1}} j_{t}}$, respectively. Plugging these solutions to the first equation and making simple calculations yields

$$
\left.v_{j_{t} j_{t}}=\left[\begin{array}{cccc}
g_{i_{t} j_{t}} & g_{i_{t} j_{t_{1}}} & \ldots & g_{i_{t} j_{t_{m}}} \\
g_{i_{t_{1}} j_{t}} & g_{i_{t_{1}} j_{t_{1}}} \ldots & g_{i_{t_{1}} j_{t_{m}}} \\
\vdots & \vdots & \vdots & \vdots \\
g_{i_{t_{m}} j_{t}} & g_{i_{t_{m}} j_{t_{1}}} \ldots & g_{i_{t_{m}} j_{t_{m}}}
\end{array}\right]^{-1} v_{j_{t_{1}} j_{t_{1}}} v_{j_{t_{2}} j_{t_{2}}} \ldots v_{j_{t_{m}} j_{t_{m}}}\right]^{-} .
$$

We should mention that the conditions $3-3$ for $j_{t}$ provide that the above determinant is different from zero.

The multiplication $(\mathrm{A}, G)\left[\begin{array}{ll}I & 0 \\ 0 & V\end{array}\right]$ gives a pair $(\mathrm{A}, H)$, where for any pair of indices $i_{t}, j_{t}, t \in T$ and for any $l=1,2, \ldots, j_{t}-1$ we have: $h_{i_{t} j_{t}}=g_{i_{t} j_{t}} v_{j_{t} j_{t}}=$ 
$g_{i_{t} j_{t}} g_{i_{t} j_{t}}^{-1}=1$ if $j_{t}=i_{t}-1$, otherwise

$$
\begin{aligned}
& h_{i_{t} j_{t}}=g_{i_{t} j_{t}} v_{j_{t} j_{t}}+g_{i_{t}\left(j_{t}+1\right)} v_{\left(j_{t}+1\right) j_{t}}+\cdots+g_{i_{t}\left(i_{t}-1\right)} v_{\left(i_{t}-1\right) j_{t}} \\
& =g_{i_{t} j_{t}} g_{i_{t} j_{t}}^{-1}\left(1-g_{i_{t}\left(j_{t}+1\right)} v_{\left(j_{t}+1\right) j_{t}}-g_{i_{t}\left(j_{t}+2\right)} v_{\left(j_{t}+2\right) j_{t}}-\cdots-g_{i_{t}\left(i_{t}-1\right)} v_{\left(i_{t}-1\right) j_{t}}\right) \\
& \quad+g_{i_{t}\left(j_{t}+1\right)} v_{\left(j_{t}+1\right) j_{t}}+\cdots+g_{i_{t}\left(i_{t}-1\right)} v_{\left(i_{t}-1\right) j_{t}}=1,
\end{aligned}
$$

and

$$
\begin{aligned}
h_{i_{t} l} & =g_{i_{t} l} v_{l l}+g_{i_{t}(l+1)} v_{(l+1) l}+\cdots+g_{i_{t}\left(i_{t}-1\right)} v_{\left(i_{t}-1\right) l} \\
= & g_{i_{t} l} v_{l l}+g_{i_{t}(l+1)} v_{(l+1) l}+\cdots+g_{i_{t}\left(j_{t}-1\right)} v_{\left(j_{t}-1\right) l}+g_{i_{t} j_{t}} v_{j_{t} l} \\
= & g_{i_{t} l} v_{l l}+g_{i_{t}(l+1)} v_{(l+1) l}+\cdots+g_{i_{t}\left(j_{t}-1\right)} v_{\left(j_{t}-1\right) l} \\
& \quad+g_{i_{t} j_{t}}\left[-g_{i_{t} j_{t}}^{-1}\left(g_{i_{t} l} v_{l l}+g_{i_{t}(l+1)} v_{(l+1) l}+\cdots+g_{i_{t}\left(j_{t}-1\right)} v_{\left(j_{t}-1\right) l}\right)\right]=0
\end{aligned}
$$

if $j_{t}=i_{t}-1$, otherwise

$$
\begin{aligned}
h_{i_{t} l}= & g_{i_{t} l} v_{l l}+g_{i_{t}(l+1)} v_{(l+1) l}+\cdots+g_{i_{t}\left(i_{t}-1\right)} v_{\left(i_{t}-1\right) l} \\
= & g_{i_{t} l} v_{l l}+g_{i_{t}(l+1)} v_{(l+1) l}+\cdots+g_{i_{t}\left(j_{t}-1\right)} v_{\left(j_{t}-1\right) l} \\
& +g_{i_{t} j_{t}} v_{j_{t} l}+g_{i_{t}\left(j_{t}+1\right)} v_{\left(j_{t}+1\right) l}+\cdots+g_{i_{t}\left(i_{t}-1\right)} v_{\left(i_{t}-1\right) l} \\
= & g_{i_{t} l} v_{l l}+g_{i_{t}(l+1)} v_{(l+1) l}+\cdots+g_{i_{t}\left(j_{t}-1\right)} v_{\left(j_{t}-1\right) l} \\
& +g_{i_{t} j_{t}}\left\{-g_{i_{t} j_{t}}^{-1}\left[\left(g_{i_{t} l} v_{l l}+g_{i_{t}(l+1)} v_{(l+1) l}+\cdots+g_{i_{t}\left(j_{t}-1\right)} v_{\left(j_{t}-1\right) l}\right)\right.\right. \\
& \left.\left.+\left(g_{i_{t}\left(j_{t}+1\right)} v_{\left(j_{t}+1\right) l}+g_{i_{t}\left(j_{t}+2\right)} v_{\left(j_{t}+2\right) l}+\cdots+g_{i_{t}\left(i_{t}-1\right)} v_{\left(i_{t}-1\right) l}\right)\right]\right\} \\
& +g_{i_{t}\left(j_{t}+1\right)} v_{\left(j_{t}+1\right) l}+g_{i_{t}\left(j_{t}+2\right)} v_{\left(j_{t}+2\right) l}+\cdots+g_{i_{t}\left(i_{t}-1\right)} v_{\left(i_{t}-1\right) l}=0 .
\end{aligned}
$$

Clearly, for any $i=1,2, \ldots, n$, and $j=1,2, \ldots, i-1$ we have: $h_{i i}=g_{i i} v_{i i}=0$, and $h_{i j}=g_{i j} v_{j j}+g_{i(j+1)} v_{(j+1) j}+\cdots+g_{i(i-1)} v_{(i-1) j}=0$ if $g_{i j}=0$ for all $j=1,2, \ldots, i-1$.

Now, we will furnish the last part of the proof. Let $K$ be a matrix of $T_{n}^{*}$ such that $k_{i i}=1$ for all $i=1,2, \ldots, n$ and

$$
k_{i i^{\prime}}=-k_{i\left(i^{\prime}+1\right)} h_{\left(i^{\prime}+1\right) j}-k_{i\left(i^{\prime}+2\right)} h_{\left(i^{\prime}+2\right) j}-\cdots-k_{i i} h_{i j}
$$

if the $j$-th column of $H$ is of the form: $h_{i^{\prime} j}=1, h_{m j}=0$ for all $m<i^{\prime}$ and $h_{i j} \neq 0$ for some $i>i^{\prime}$. Then $K(\mathrm{~A}, H)=(\mathrm{A}, L)$, where

$$
\begin{aligned}
l_{i j} & =k_{i(j+1)} h_{(j+1) j}+k_{i(j+2)} h_{(j+2) j}+\cdots+k_{i i} h_{i j} \\
& =k_{i i^{\prime}}+k_{i\left(i^{\prime}+1\right)} h_{\left(i^{\prime}+1\right) j}+k_{i\left(i^{\prime}+2\right)} h_{\left(i^{\prime}+2\right) j}+\cdots+k_{i i} h_{i j}=0 .
\end{aligned}
$$

Therefore, $(\mathrm{A}, L)=(\mathrm{A}, \mathrm{B})$, which completes the proof.

Corollary 1. The total number of $G L_{2}\left(T_{n}\right)$-orbits is equal to the number of pairs $(\mathrm{A}, \mathrm{B}) \in{ }^{2} T_{n}$. 
Example 1. Consider the pair

$(\mathrm{A}, G)=\left(\left[\begin{array}{lllllll}1 & 0 & 0 & 0 & 0 & 0 & 0 \\ 0 & 1 & 0 & 0 & 0 & 0 & 0 \\ 0 & 0 & 0 & 0 & 0 & 0 & 0 \\ 0 & 0 & 0 & 1 & 0 & 0 & 0 \\ 0 & 0 & 0 & 0 & 0 & 0 & 0 \\ 0 & 0 & 0 & 0 & 0 & 0 & 0 \\ 0 & 0 & 0 & 0 & 0 & 0 & 0\end{array}\right],\left[\begin{array}{ccccccc}0 & 0 & 0 & 0 & 0 & 0 & 0 \\ 0 & 0 & 0 & 0 & 0 & 0 & 0 \\ g_{31} & g_{32} & 0 & 0 & 0 & 0 & 0 \\ 0 & 0 & 0 & 0 & 0 & 0 & 0 \\ g_{51} & g_{52} & g_{53} & g_{54} & 0 & 0 & 0 \\ g_{61} & g_{62} & g_{63} & g_{64} & 0 & 0 & 0 \\ g_{71} & g_{72} & g_{73} & g_{74} & 0 & 0 & 0\end{array}\right]\right) \in{ }^{2} T_{7}$

where $g_{32}, g_{54}, g_{63}, g_{71} \in F^{*}$. Based on the proof of Theorem 2 we find the indices $i_{t}, j_{t}, t \in T$ and determine the corresponding entries of $V \in T_{7}^{*}$ :

- $i_{1}=3, j_{1}=2$,

$v_{22}=g_{32}^{-1}$,

$v_{21}=-g_{32}^{-1} g_{31} v_{11}$;

- $i_{2}=5, j_{2}=4$,

$v_{44}=g_{54}^{-1}$,

$v_{41}=-g_{54}^{-1}\left(g_{51} v_{11}+g_{52} v_{21}+g_{53} v_{31}\right)$,

$v_{42}=-g_{54}^{-1}\left(g_{52} v_{22}+g_{53} v_{32}\right)$,

$v_{43}=-g_{54}^{-1} g_{53} v_{33}$;

- $i_{3}=6, j_{3}=3$,

$v_{33}=g_{63}^{-1}\left(1-g_{64} v_{43}\right)$,

$v_{31}=-g_{63}^{-1}\left(g_{61} v_{11}+g_{62} v_{21}+g_{64} v_{41}\right)$,

$v_{32}=-g_{63}^{-1}\left(g_{62} v_{22}+g_{64} v_{42}\right)$;

- $i_{4}=7, j_{4}=1$,

$v_{11}=g_{71}^{-1}\left(1-g_{72} v_{21}-g_{73} v_{31}-g_{74} v_{41}\right)$.

The result of multiplication $(\mathrm{A}, G)\left[\begin{array}{cc}I & 0 \\ 0 & V\end{array}\right]$ is a pair $(\mathrm{A}, H) \in{ }^{2} T_{7}$, where: $h_{32}=g_{32} v_{22}=g_{32} g_{32}^{-1}=1, h_{31}=g_{31} v_{11}+g_{32} v_{21}=g_{31} v_{11}+g_{32}\left(-g_{32}^{-1} g_{31} v_{11}\right)=$ $=0$,

$h_{54}=g_{54} v_{44}=g_{54} g_{54}^{-1}=1, h_{51}=g_{51} v_{11}+g_{52} v_{21}+g_{53} v_{31}+g_{54} v_{41}=g_{51} v_{11}+$ $+g_{52} v_{21}+g_{53} v_{31}+g_{54}\left[-g_{54}^{-1}\left(g_{51} v_{11}+g_{52} v_{21}+g_{53} v_{31}\right)\right]=0, h_{52}=g_{52} v_{22}+$ $+g_{53} v_{32}+g_{54} v_{42}=g_{52} v_{22}+g_{53} v_{32}+g_{54}\left[-g_{54}^{-1}\left(g_{52} v_{22}+g_{53} v_{32}\right)\right]=0$,

$h_{53}=g_{53} v_{33}+g_{54} v_{43}=g_{53} v_{33}+g_{54}\left(-g_{54}^{-1} g_{53} v_{33}\right)=0$,

$h_{63}=g_{63} v_{33}+g_{64} v_{43}=g_{63} g_{63}^{-1}\left(1-g_{64} v_{43}\right)+g_{64} v_{43}=1, h_{61}=g_{61} v_{11}+g_{62} v_{21}+$ $+g_{63} v_{31}+g_{64} v_{41}=g_{61} v_{11}+g_{62} v_{21}+g_{63}\left[-g_{63}^{-1}\left(g_{61} v_{11}+g_{62} v_{21}+g_{64} v_{41}\right)\right]+$ $+g_{64} v_{41}=0, h_{62}=g_{62} v_{22}+g_{63} v_{32}+g_{64} v_{42}=g_{62} v_{22}+g_{63}\left[-g_{63}^{-1}\left(g_{62} v_{22}+g_{64} v_{42}\right)\right]+$ $+g_{64} v_{42}=0$,

$h_{71}=g_{71} v_{11}+g_{72} v_{21}+g_{73} v_{31}+g_{74} v_{41}=g_{71} g_{71}^{-1}\left(1-g_{72} v_{21}-g_{73} v_{31}-g_{74} v_{41}\right)+$ $+g_{72} v_{21}+g_{73} v_{31}+g_{74} v_{41}=1$.

We have $h_{i i}=g_{i i} v_{i i}=0$ for any $i=1,2, \ldots, 7$ and $h_{21}=g_{21} v_{11}=0$, $h_{4 j}=g_{4 j} v_{j j}+g_{4(j+1)} v_{(j+1) j}+\cdots+g_{43} v_{3 j}=0$ for $j=1,2,3$. Moreover, 
$h_{65}=g_{65} v_{55}=0, h_{75}=g_{75} v_{55}+g_{76} v_{65}=0, h_{76}=g_{76} v_{66}=0$. Thus

$$
H=\left[\begin{array}{ccccccc}
0 & 0 & 0 & 0 & 0 & 0 & 0 \\
0 & 0 & 0 & 0 & 0 & 0 & 0 \\
0 & 1 & 0 & 0 & 0 & 0 & 0 \\
0 & 0 & 0 & 0 & 0 & 0 & 0 \\
0 & 0 & 0 & 1 & 0 & 0 & 0 \\
0 & 0 & 1 & h_{64} & 0 & 0 & 0 \\
1 & h_{72} & h_{73} & h_{74} & 0 & 0 & 0
\end{array}\right] .
$$

Then $K(\mathrm{~A}, H)=(\mathrm{A}, \mathrm{B})$, where

$$
\begin{aligned}
& K=\left[\begin{array}{cccccll}
1 & 0 & 0 & 0 & 0 & 0 & 0 \\
0 & 1 & 0 & 0 & 0 & 0 & 0 \\
0 & 0 & 1 & 0 & 0 & 0 & 0 \\
0 & 0 & 0 & 1 & 0 & 0 & 0 \\
0 & 0 & 0 & 0 & 1 & 0 & 0 \\
0 & 0 & 0 & 0 & -h_{64} & 0 & 0 \\
0 & 0 & -h_{72} & 0 & h_{73} h_{64}-h_{74} & -h_{73} & 1
\end{array}\right] \\
& \text { and }(A, B)=\left(\left[\begin{array}{lllllll}
1 & 0 & 0 & 0 & 0 & 0 & 0 \\
0 & 1 & 0 & 0 & 0 & 0 & 0 \\
0 & 0 & 0 & 0 & 0 & 0 & 0 \\
0 & 0 & 0 & 1 & 0 & 0 & 0 \\
0 & 0 & 0 & 0 & 0 & 0 & 0 \\
0 & 0 & 0 & 0 & 0 & 0 & 0 \\
0 & 0 & 0 & 0 & 0 & 0 & 0
\end{array}\right],\left[\begin{array}{lllllll}
0 & 0 & 0 & 0 & 0 & 0 & 0 \\
0 & 0 & 0 & 0 & 0 & 0 & 0 \\
0 & 1 & 0 & 0 & 0 & 0 & 0 \\
0 & 0 & 0 & 0 & 0 & 0 & 0 \\
0 & 0 & 0 & 1 & 0 & 0 & 0 \\
0 & 0 & 1 & 0 & 0 & 0 & 0 \\
1 & 0 & 0 & 0 & 0 & 0 & 0
\end{array}\right]\right) \text {. }
\end{aligned}
$$

Theorem 3. The total number of $G L_{2}\left(T_{n}\right)$-orbits is equal to the Bell number $B_{n}$.

Proof. Recall that the Bell number $B_{n}$ is the number of partitions $\Pi$ of the set $\{1,2, \ldots n\}$. Therefore, in the light of Corollary 1 it suffices to show that the set of pairs (A,B) $\in{ }^{2} T_{n}$ and the family of partitions $\Pi$ are equipotent.

Let $A_{k}$ be the matrix obtained from $\mathrm{A}=\left(a_{k l}\right)$ by removing its rows and columns number $k+1, k+2, \ldots, n$ if $k=1,2, \ldots, n-1$, and let $A_{k}=\mathrm{A}$ if $k=n$. The rank of a matrix $A_{k}$ is denoted by $t_{k}$ and the expression $T$ means the set $\left\{t_{k} ; k=1,2, \ldots, n\right\}$. There exists a bijection that associates to every pair $(\mathrm{A}, \mathrm{B}) \in{ }^{2} T_{n}$ the partition $\Pi=\left\{U_{t_{k}}, t_{k} \in T\right\}$ such that for any $k \in\{1,2, \ldots n\}$ with $a_{k k}=1$

$$
U_{t_{k}}=\{k\} \cup\left\{i ; b_{i j}=1, t_{k}=j-\operatorname{rank} B_{i j}, 1 \leqslant i \leqslant n, 1 \leqslant j \leqslant n\right\},
$$

where $B_{i j}$ is the matrix obtained from $\mathrm{B}=\left(b_{i j}\right)$ by changing its rows number $i, i+1, \ldots, n$ and columns number $j, j+1, \ldots, n$ into zero rows and columns.

Conversely, to a partition $\Pi=\left\{U_{s}, s \in S\right\}$ of the set $\{1,2, \ldots n\}$ corresponds exactly one pair $(\mathrm{A}, \mathrm{B}) \in{ }^{2} T_{n}$. To see this correspondence, suppose that $u_{s}$ is the smallest element of the set $U_{s}$, where $s \in S$. An entry $a_{i j}$ of $\mathrm{A}=\left(\mathrm{a}_{\mathrm{ij}}\right)$ is equal to 1 if $i=j=u_{s}$ for some $s \in S$, being equal to 0 otherwise. 
Elements of the set

$$
\{1,2, \ldots n\} \backslash\left\{u_{s}, s \in S\right\}=\left\{x_{m} ; m=1,2, \ldots, n-\operatorname{rank} \mathrm{A}\right\},
$$

where $x_{1}<x_{2}<\cdots<x_{n-\text { rankA }}$, are numbers of rows with entry 1 of the matrix B. Any other row of B is a zero row. We have to indicate the column number $y_{m}$ containing entry 1 from a row $x_{m}$ for any $m=1,2, \ldots, n-\operatorname{rank}$. We do it for each row one by one, from the smallest $x_{m}$, i.e. $x_{1}$, to the greatest, i.e. $x_{n-\operatorname{rank\mathrm {A}}}$. This order is crucial. For any $m=1,2, \ldots, n-\operatorname{rank\mathrm {A}}$ the column $y_{m}$ is the $s$-th column without entry 1 (from the left). Thereby, entry 1 in the $x_{1}$-th row is in the $s$-th column, where $x_{1} \in U_{s}$.

Corollary 2. The orbit of unimodular cyclic submodules of ${ }^{2} T_{n}$ represented by the pair $(1,0)$ of ${ }^{2} T_{n}$ corresponds to the partition $\Pi=\{\{1\},\{2\}, \ldots,\{n\}\}$ of the set $\{1,2, \ldots n\}$.

Example 2. Choose the pair

$$
(\mathrm{A}, \mathrm{B})=\left(\left[\begin{array}{llllll}
1 & 0 & 0 & 0 & 0 & 0 \\
0 & 1 & 0 & 0 & 0 & 0 \\
0 & 0 & 0 & 0 & 0 & 0 \\
0 & 0 & 0 & 1 & 0 & 0 \\
0 & 0 & 0 & 0 & 0 & 0 \\
0 & 0 & 0 & 0 & 0 & 0
\end{array}\right],\left[\begin{array}{llllll}
0 & 0 & 0 & 0 & 0 & 0 \\
0 & 0 & 0 & 0 & 0 & 0 \\
0 & 1 & 0 & 0 & 0 & 0 \\
0 & 0 & 0 & 0 & 0 & 0 \\
0 & 0 & 0 & 1 & 0 & 0 \\
0 & 0 & 1 & 0 & 0 & 0
\end{array}\right]\right) \in{ }^{2} T_{6} .
$$

We have $a_{k k}=1$ for $k=1,2,4$, so $\Pi=\left\{U_{t_{1}}, U_{t_{2}}, U_{t_{4}}\right\}$, where $t_{1}=1, t_{2}=2$, $t_{4}=3$. Of course, $1 \in U_{1}, 2 \in U_{2}, 4 \in U_{3}$. Moreover,

- $b_{32}=1$, thus $j-\operatorname{rank} B_{32}=2-0=2$ and hence $3 \in U_{2}$;

- $b_{54}=1$, thus $j-\operatorname{rank} B_{54}=4-1=3$ and hence $5 \in U_{3}$;

- $b_{63}=1$, thus $j-\operatorname{rank} B_{63}=3-1=2$ and hence $6 \in U_{2}$.

Therefore, the partition $\Pi=\{\{1\},\{2,3,6\},\{4,5\}\}$ corresponds to such a pair $(\mathrm{A}, \mathrm{B})$.

We will also show the converse correspondence. As $\left\{u_{s}, s \in S\right\}=\{1,2,4\}$, so $a_{11}=a_{22}=a_{44}=1$ and all the remaining entries of $\mathrm{A}$ are zero. Moreover, $x_{1}=3, x_{2}=5, x_{3}=6$, hence rows 3,5 and 6 of B contain entry $1.3 \in U_{2}$, thus $b_{32}=1.5 \in U_{3}$ and the 3rd column without entry 1 (from the left) is now the column number 4 , therefore $b_{54}=1$. By the same way we get that $b_{63}=1$, all the remaining entries of $\mathrm{B}$ being zero. Finally, the pair of ${ }^{2} T_{6}$ corresponding to $\Pi$ is equal to $(\mathrm{A}, \mathrm{B})$.

Example 3. Consider the ring $T_{4}$. By Theorem 3 there are exactly $B_{4}=15$ $G L_{2}\left(T_{4}\right)$-orbits with the following representatives and corresponding partitions of the set $\{1,2,3,4\}$ :

$$
T_{4}\left(\left[\begin{array}{llll}
1 & 0 & 0 & 0 \\
0 & 1 & 0 & 0 \\
0 & 0 & 1 & 0 \\
0 & 0 & 0 & 1
\end{array}\right],\left[\begin{array}{llll}
0 & 0 & 0 & 0 \\
0 & 0 & 0 & 0 \\
0 & 0 & 0 & 0 \\
0 & 0 & 0 & 0
\end{array}\right]\right), \Pi=\{\{1\},\{2\},\{3\},\{4\}\} ;
$$


$T_{4}\left(\left[\begin{array}{cccc}1 & 0 & 0 & 0 \\ 0 & 1 & 0 & 0 \\ 0 & 0 & 1 & 0 \\ 0 & 0 & 0 & 0\end{array}\right],\left[\begin{array}{cccc}0 & 0 & 0 & 0 \\ 0 & 0 & 0 & 0 \\ 0 & 0 & 0 & 0 \\ 1 & 0 & 0 & 0\end{array}\right]\right), \Pi=\{\{1,4\},\{2\},\{3\}\} ;$

$T_{4}\left(\left[\begin{array}{llll}1 & 0 & 0 & 0 \\ 0 & 1 & 0 & 0 \\ 0 & 0 & 1 & 0 \\ 0 & 0 & 0 & 0\end{array}\right],\left[\begin{array}{cccc}0 & 0 & 0 & 0 \\ 0 & 0 & 0 & 0 \\ 0 & 0 & 0 & 0 \\ 0 & 1 & 0 & 0\end{array}\right]\right), \Pi=\{\{1\},\{2,4\},\{3\}\} ;$

$T_{4}\left(\left[\begin{array}{llll}1 & 0 & 0 & 0 \\ 0 & 1 & 0 & 0 \\ 0 & 0 & 1 & 0 \\ 0 & 0 & 0 & 0\end{array}\right],\left[\begin{array}{llll}0 & 0 & 0 & 0 \\ 0 & 0 & 0 & 0 \\ 0 & 0 & 0 & 0 \\ 0 & 0 & 1 & 0\end{array}\right]\right), \Pi=\{\{1\},\{2\},\{3,4\}\} ;$

$T_{4}\left(\left[\begin{array}{llll}1 & 0 & 0 & 0 \\ 0 & 1 & 0 & 0 \\ 0 & 0 & 0 & 0 \\ 0 & 0 & 0 & 1\end{array}\right],\left[\begin{array}{llll}0 & 0 & 0 & 0 \\ 0 & 0 & 0 & 0 \\ 1 & 0 & 0 & 0 \\ 0 & 0 & 0 & 0\end{array}\right]\right), \Pi=\{\{1,3\},\{2\},\{4\}\} ;$

$T_{4}\left(\left[\begin{array}{llll}1 & 0 & 0 & 0 \\ 0 & 1 & 0 & 0 \\ 0 & 0 & 0 & 0 \\ 0 & 0 & 0 & 1\end{array}\right],\left[\begin{array}{cccc}0 & 0 & 0 & 0 \\ 0 & 0 & 0 & 0 \\ 0 & 1 & 0 & 0 \\ 0 & 0 & 0 & 0\end{array}\right]\right), \Pi=\{\{1\},\{2,3\},\{4\}\} ;$

$T_{4}\left(\left[\begin{array}{llll}1 & 0 & 0 & 0 \\ 0 & 0 & 0 & 0 \\ 0 & 0 & 1 & 0 \\ 0 & 0 & 0 & 1\end{array}\right],\left[\begin{array}{cccc}0 & 0 & 0 & 0 \\ 1 & 0 & 0 & 0 \\ 0 & 0 & 0 & 0 \\ 0 & 0 & 0 & 0\end{array}\right]\right), \Pi=\{\{1,2\},\{3\},\{4\}\} ;$

$T_{4}\left(\left[\begin{array}{llll}1 & 0 & 0 & 0 \\ 0 & 1 & 0 & 0 \\ 0 & 0 & 0 & 0 \\ 0 & 0 & 0 & 0\end{array}\right],\left[\begin{array}{llll}0 & 0 & 0 & 0 \\ 0 & 0 & 0 & 0 \\ 1 & 0 & 0 & 0 \\ 0 & 1 & 0 & 0\end{array}\right]\right), \Pi=\{\{1,3,4\},\{2\}\} ;$

$T_{4}\left(\left[\begin{array}{llll}1 & 0 & 0 & 0 \\ 0 & 1 & 0 & 0 \\ 0 & 0 & 0 & 0 \\ 0 & 0 & 0 & 0\end{array}\right],\left[\begin{array}{llll}0 & 0 & 0 & 0 \\ 0 & 0 & 0 & 0 \\ 1 & 0 & 0 & 0 \\ 0 & 0 & 1 & 0\end{array}\right]\right), \Pi=\{\{1,3\},\{2,4\}\} ;$

$T_{4}\left(\left[\begin{array}{llll}1 & 0 & 0 & 0 \\ 0 & 1 & 0 & 0 \\ 0 & 0 & 0 & 0 \\ 0 & 0 & 0 & 0\end{array}\right],\left[\begin{array}{llll}0 & 0 & 0 & 0 \\ 0 & 0 & 0 & 0 \\ 0 & 1 & 0 & 0 \\ 1 & 0 & 0 & 0\end{array}\right]\right), \Pi=\{\{1,4\},\{2,3\}\} ;$

$T_{4}\left(\left[\begin{array}{llll}1 & 0 & 0 & 0 \\ 0 & 1 & 0 & 0 \\ 0 & 0 & 0 & 0 \\ 0 & 0 & 0 & 0\end{array}\right],\left[\begin{array}{llll}0 & 0 & 0 & 0 \\ 0 & 0 & 0 & 0 \\ 0 & 1 & 0 & 0 \\ 0 & 0 & 1 & 0\end{array}\right]\right), \Pi=\{\{1\},\{2,3,4\}\} ;$ 


$$
\begin{aligned}
& T_{4}\left(\left[\begin{array}{llll}
1 & 0 & 0 & 0 \\
0 & 0 & 0 & 0 \\
0 & 0 & 1 & 0 \\
0 & 0 & 0 & 0
\end{array}\right],\left[\begin{array}{llll}
0 & 0 & 0 & 0 \\
1 & 0 & 0 & 0 \\
0 & 0 & 0 & 0 \\
0 & 1 & 0 & 0
\end{array}\right]\right), \Pi=\{\{1,2,4\},\{3\}\} \\
& T_{4}\left(\left[\begin{array}{llll}
1 & 0 & 0 & 0 \\
0 & 0 & 0 & 0 \\
0 & 0 & 1 & 0 \\
0 & 0 & 0 & 0
\end{array}\right],\left[\begin{array}{cccc}
0 & 0 & 0 & 0 \\
1 & 0 & 0 & 0 \\
0 & 0 & 0 & 0 \\
0 & 0 & 1 & 0
\end{array}\right]\right), \Pi=\{\{1,2\},\{3,4\}\} ; \\
& T_{4}\left(\left[\begin{array}{llll}
1 & 0 & 0 & 0 \\
0 & 0 & 0 & 0 \\
0 & 0 & 0 & 0 \\
0 & 0 & 0 & 1
\end{array}\right],\left[\begin{array}{llll}
0 & 0 & 0 & 0 \\
1 & 0 & 0 & 0 \\
0 & 1 & 0 & 0 \\
0 & 0 & 0 & 0
\end{array}\right]\right), \Pi=\{\{1,2,3\},\{4\}\} ; \\
& T_{4}\left(\left[\begin{array}{llll}
1 & 0 & 0 & 0 \\
0 & 0 & 0 & 0 \\
0 & 0 & 0 & 0 \\
0 & 0 & 0 & 0
\end{array}\right],\left[\begin{array}{llll}
0 & 0 & 0 & 0 \\
1 & 0 & 0 & 0 \\
0 & 1 & 0 & 0 \\
0 & 0 & 1 & 0
\end{array}\right]\right), \Pi=\{\{1,2,3,4\}\} .
\end{aligned}
$$

Open Access. This article is licensed under a Creative Commons Attribution 4.0 International License, which permits use, sharing, adaptation, distribution and reproduction in any medium or format, as long as you give appropriate credit to the original author(s) and the source, provide a link to the Creative Commons licence, and indicate if changes were made. The images or other third party material in this article are included in the article's Creative Commons licence, unless indicated otherwise in a credit line to the material. If material is not included in the article's Creative Commons licence and your intended use is not permitted by statutory regulation or exceeds the permitted use, you will need to obtain permission directly from the copyright holder. To view a copy of this licence, visit http://creativecommons. org/licenses/by/4.0/.

\section{References}

[1] Bartnicka, E., Matraś, A.: Free cyclic submodules in the context of the projective line. Results Math. 70, 567-580 (2016)

[2] Bartnicka, E., Matraś, A.: The distant graph of the projective line over a finite ring with unity. Results Math. 72, 1943-1958 (2017)

[3] Bartnicka, E., Saniga, M.: Affine and projective planes linked with projective lines over certain rings of lower triangular matrices. Linear Algebra Appl. 586, 377-409 (2020)

[4] Blunck, A., Havlicek, H.: Projective Representations I: Projective lines over rings. Abh. Math. Sem. Univ. Hamburg 70, 287-299 (2000)

[5] Havlicek, H.: Divisible designs, laguerre geometry, and beyond. J. Math. Sci. New York 186, 882-926 (2012) 
[6] Havlicek, H., Saniga, M.: Vectors, Cyclic Submodules and Projective Spaces Linked with Ternions. J. Geometry 92(1-2), 79-90 (2009)

[7] Herzer, A.: Chain Geometries. In: Buekenhout, F. (ed.) Handbook of Incidence Geometry, pp. 781-842. Elsevier, Amsterdam (1995)

[8] Saniga, M., Havlicek, H., Planat, M., Pracna, P.: Twin 'Fano-Snowflakes' over the Smallest Ring of Ternions, Symmetry, Integrability and Geometry: Methods and Applications, Vol. 4, Paper 050, p. 7 (2008)

Edyta Bartnicka

Institute of Information Technology

Warsaw University of Life Sciences - SGGW

Warsaw

Poland

e-mail: edyta_bartnicka@sggw.edu.pl

Received: December 19, 2020.

Accepted: April 16, 2021.

Publisher's Note Springer Nature remains neutral with regard to jurisdictional claims in published maps and institutional affiliations. 